\title{
GABAergic Excitatory Synapses and Electrical Coupling Sustain Prolonged Discharges in the Prey Capture Neural Network of Clione limacina
}

\author{
Tigran P. Norekian \\ Department of Biology, Arizona State University, Tempe, Arizona 85287-1501, and Friday Harbor Laboratories, University \\ of Washington, Friday Harbor, Washington 98250
}

\begin{abstract}
Afterdischarges represent a prominent characteristic of the neural network that controls prey capture reactions in the carnivorous mollusc Clione limacina. Their main functional implication is transformation of a brief sensory input from a prey into a lasting prey capture response. The present study, which focuses on the neuronal mechanisms of afterdischarges, demonstrates that a single pair of interneurons [cerebral A interneuron (Cr-Aint)] is responsible for afterdischarge generation in the network. Cr-Aint neurons are electrically coupled to all other neurons in the network and produce slow excitatory synaptic inputs to them. This excitatory transmission is found to be GABAergic, which is demonstrated by the use of GABA antagonists, uptake inhibitors, and double-labeling experiments showing that Cr-Aint neurons are GABA-immunoreactive. The Cr-Aint neurons organize three different pathways in the prey capture network, which provide positive feedback necessary
\end{abstract}

for sustaining prolonged spike activity. The first pathway includes electrical coupling and slow chemical transmission from the Cr-Aint neurons to all other neurons in the network. The second feedback is based on excitatory reciprocal connections between contralateral interneurons. Recurrent excitation via the contralateral cell can sustain prolonged interneuron firing, which then drives the activity of all other cells in the network. The third positive feedback is represented by prominent afterdepolarizing potentials after individual spikes in the Cr-Aint neurons. Afterdepolarizations apparently represent recurrent GABAergic excitatory inputs. It is suggested here that these afterdepolarizing potentials are produced by GABAergic excitatory autapses.

Key words: recurrent inputs; autapses; afterdepolarization; afterdischarge; positive feedback; neural network; mollusc; feeding; GABAergic interneuron; GABA immunoreactivity
Neuronal afterdischarges represent an essential and widespread phenomenon in brain functioning. The main important implication of afterdischarges during signal processing in the brain is a transformation of a brief input to the network into a lasting output. Amplification of the feedforward signal during afterdischarges also carries a significant functional role in many systems (ter Maat et al., 1988; Brown and Mayeri, 1989; Douglas et al., 1995). Mechanisms of afterdischarge generation could be similar in many different systems, and, therefore, their understanding in one experimental model will have a broad theoretical value.

Generation of prolonged afterdischarges was found to be a prominent characteristic of the neural network, which controls prey capture reactions in the carnivorous mollusc Clione limacina (Norekian, 1993). Clione is a highly specialized predator that has adapted feeding structures for prey capture: oral appendages, called buccal cones (Wagner, 1885; Lalli, 1970). Buccal cones are normally cone-shaped and retracted inside the buccal cavity. Contact with a prey triggers fast protraction of the buccal cones, which then become tentacle-like and grasp the prey. Sometimes, Clione misses the prey and continues to swim in search of it with fully protracted buccal cones for several seconds after an unsuccessful first strike. Long-lasting protraction of the buccal cones is especially typical when Clione is aroused in the vicinity of other

\footnotetext{
Received Nov. 3, 1998; revised Dec. 15, 1998; accepted Dec. 18, 1998.

This study was supported by National Institute of Neurological Disorders and Stroke Grant NS-34662 and National Science Foundation Grant IBN-9630805.

Correspondence should be addressed to Dr. Tigran Norekian, Department of Biology, Arizona State University, Tempe, AZ 85287-1501.

Copyright (C) 1999 Society for Neuroscience $\quad 0270-6474 / 99 / 191863-13 \$ 05.00 / 0$
}

feeding animals (Litvinova and Orlovsky, 1985). The prey capture neural network includes at least 26 electrically coupled cerebral motoneurons [cerebral A motoneuron ( $\mathrm{Cr}-\mathrm{A})$ ] and a bilaterally symmetrical interneuron [cerebral A interneuron (Cr-Aint)] that organizes coordination between contralateral Cr-A neurons (Arshavsky et al., 1993; Norekian and Satterlie, 1993a). Discharges, which can be triggered in the normally silent network by brief sensory inputs or induced bursts of spikes in a single cell, last up to $30 \mathrm{sec}$ and play two major roles in the functioning of the Cr-A neuron network (Norekian, 1993). First, they transfer a short burst of activity into a prolonged motoneuron discharge, and thereby into a lasting behavioral response-protraction of the prey capture appendages. Second, amplification of a signal during afterdischarge ensures the recruitment of all $\mathrm{Cr}-\mathrm{A}$ neurons into firing activity, which is necessary for producing a normal prey capture reaction.

The current investigation focuses on the mechanisms that underlie afterdischarge generation in the population of $\mathrm{Cr}-\mathrm{A}$ neurons in Clione. Preliminary experiments demonstrated that individual $\mathrm{Cr}-\mathrm{A}$ motoneurons are incapable of producing afterdischarges, and synaptic excitation is the most probable mechanism (Norekian, 1993). Here, it is shown that a pair of Cr-Aint neurons is necessary for afterdischarge generation. CrAint neurons organize all positive feedbacks, which sustain prolonged neuron firing in the network. Each $\mathrm{Cr}$-Aint neuron produces GABAergic excitatory synapses to all $\mathrm{Cr}$-A motoneurons and is electrically coupled to them, thus creating one positive feedback mechanism. Each Cr-Aint neuron also produces excitatory synaptic inputs to its contralateral compatriot, thus providing 
a pathway for recurrent excitation via the contralateral cell. The third possible feedback suggested by the present investigation is the existence of recurrent synapses produced by Cr-Aint neurons on themselves ("autapses"; Van der Loos and Glaser, 1972).

\section{MATERIALS AND METHODS}

Preparation. Adult specimens of Clione limacina, body length of $1-3 \mathrm{~cm}$, were collected from the breakwater at Friday Harbor Laboratories, University of Washington (Friday Harbor, WA) in the Spring-Summer season and were held in 1-gallon jars in a refrigerator at $5-7^{\circ} \mathrm{C}$. Animals were anesthetized in a 1:1 mixture of seawater and isotonic $\mathrm{MgCl}_{2}$ and dissected in a Sylgard (Dow Corning, Midland, MI)-coated Petri dish. Electrophysiological experiments were performed on reduced preparations consisting of the CNS, head, and wings. All nerves running from the central ganglia to the head and wings remained intact. Before electrophysiological recordings, the sheaths of the central ganglia were soften by bathing the preparation in a $1 \mathrm{mg} / \mathrm{ml}$ solution of protease (type XIV; Sigma, St. Louis, MO) for $\sim 5 \mathrm{~min}$, followed by a $30 \mathrm{~min}$ wash.

Electrophysiological recordings. Intracellular recordings were made with glass microelectrodes filled with $2 \mathrm{M}$ potassium acetate. Microelectrodes had resistances of 10-30 M . Electrophysiological signals were amplified, displayed, and recorded by using standard electrophysiological techniques. Intracellular stimulation was provided via amplifier bridge circuit, which was balanced before each experiment. Changes in the membrane potential during measurements of electrical coupling or reversal potentials were provided by using two electrodes in one cell: one for current injections and the other for voltage recording. Changes in the membrane conductance were estimated by measuring changes in the amplitude of the membrane potential hyperpolarization steps, which were induced by injecting constant negative current pulses via a second intracellular electrode. Individual neurons were identified based on their electrophysiological properties, morphology, and motor effects. To test for monosynaptic connections, a high- $\mathrm{Mg}^{2+} /$ high- $\mathrm{Ca}^{2+}$ solution $(2.5 \times$ normal; $110 \mathrm{~mm} \mathrm{MgCl}_{2}$ and $25 \mathrm{mM} \mathrm{CaCl}_{2}$ ) was used. High divalent solutions work appropriately in Clione preparations (for example, see Huang and Satterlie, 1990). Intracellular staining of neurons was achieved via recording electrodes filled with a $5 \%$ solution of neurobiotin (Vector Laboratories, Burlingame, CA). Neurobiotin was iontophoresed by applying positive current pulses of $+2 \mathrm{nA}$ amplitude for 20-30 min. After fixation in $4 \%$ paraformaldehyde and $0.1 \%$ glutaraldehyde in PBS, preparations were incubated $12 \mathrm{hr}$ in fluorescein-labeled avidin (Vector Laboratories) to visualize neurobiotin-filled cells.

Reagents and drugs. GABA, its antagonists 5-aminovaleric acid, saclofen, phaclofen, piperidine-4-sulfonic acid (piperidine-4-S) (all from Research Biochemicals, Natick, MA), bicuculline methchloride, picrotoxin (both from Sigma), and uptake inhibitor nipecotic acid (Research Biochemicals) were applied with the use of a graduated $1 \mathrm{ml}$ pipette. The final concentration was estimated from the known volume of injected solution and the known volume of saline in the recording dish. The duration and the firing frequency of induced bursts of spikes in interneurons were constant in the controls and during drug application, when effects of the drugs on the postsynaptic responses were studied (duration was usually $0.5 \mathrm{sec}$; the firing frequency varied in different experiments from 10 to $20 \mathrm{~Hz}$ ). To test whether a particular neuron was directly sensitive to GABA, neurons were chemically isolated by a high- $\mathrm{Mg}^{2+}$ solution consisting of a 1:3 mix of $0.33 \mathrm{M} \mathrm{MgCl}_{2}$ in seawater superfused into the preparation dish.

$\mathrm{Na}^{+}$-replaced solution. Filtered natural seawater was used in most experiments. Artificial seawater composition was (in $\mathrm{mm}$ ): $\mathrm{NaCl} 420$, $\mathrm{MgSO}_{4} 25, \mathrm{MgCl}_{2} 25, \mathrm{KCl} 10, \mathrm{CaCl}_{2} 10$, and $\mathrm{NaHCO}_{3} 2, \mathrm{pH}$ adjusted to 7.4. $\mathrm{Na}^{+}$-replaced saline was made by substituting with L-arginine hydrochloride (Sigma) on an equimolar basis. Ten normality $\mathrm{NaOH}$ was used to adjust $\mathrm{pH}$ to 7.4; therefore, the final concentration of $\mathrm{Na}^{+}$was $\sim 2 \%$. Four hundred micromolar L-arginine hydrochloride in normal seawater did not have an essential effect on the Cr-A neuron activity, only slightly depolarizing their membrane potential (not more than $5 \mathrm{mV} ; n=$ 4). It also did not interfere with the effect of GABA on the Cr-A neurons $(n=4)$. Replacement of $\mathrm{Ca}^{2+}$ in zero- $\mathrm{Ca}^{2+}$ saline was done by substituting $\mathrm{Mg}^{2+}$.

GABA-immunocytochemistry and double-labeling experiments. Because Clione limacina is a relatively small mollusc, entire reduced preparations were used for the whole-mount immunocytochemical procedure. The preparations were fixed for $3 \mathrm{hr}$ in $4 \%$ paraformaldehyde and $0.1 \%$ glutaraldehyde in PBS ( $\mathrm{pH} 6.5-7.0)$ at room temperature $\left(15-20^{\circ} \mathrm{C}\right)$. To reduce high nonspecific fluorescence caused by glutaraldehyde fixation, the tissues were incubated overnight in $4 \%$ sodium borohydride in PBS (Kosaka et al., 1986). The preparations were then transferred to $3 \mathrm{ml}$ vials, where they were washed for $12 \mathrm{hr}$ in PBS and preincubated in PBS containing $0.1 \%$ Triton X-100 to increase tissue permeability. The tissues were then exposed to $6 \%$ goat serum in PBS and $0.1 \%$ Triton $\mathrm{X}-100$ for $6 \mathrm{hr}$ to reduce nonspecific staining and incubated $36 \mathrm{hr}$ at $5^{\circ} \mathrm{C}$ in GABA antibody (polyclonal GABA antisera raised in guinea pig; Eugene Tech International, Inc., Ridgefield Park, NJ). The dilution of primary antibody was 1:500. After a $12 \mathrm{hr}$ wash in PBS, the tissues were placed for 24 $\mathrm{hr}$ in fluorescein-labeled secondary antibody (working concentration of $40 \mu \mathrm{g} / \mathrm{ml}$; anti-guinea pig IgG produced in goat; Vector Laboratories). The secondary antibody was removed with several PBS exchanges, and preparations were washed overnight. The tissues were then cleared in xylene, mounted in DPX, and examined in whole mount with a Nikon (Tokyo, Japan) fluorescence microscope and a Bio-Rad (Hercules, CA) MRC 600 laser scanning confocal microscope. The first set of the control experiments included preadsorption of the primary antibody with $50 \mu \mathrm{M}$ GABA-BSA conjugate for $6 \mathrm{hr}$ before processing the tissue. In the second set of controls, primary antibody was omitted from the procedure. No staining resulted in both sets of control experiments.

For double-labeling experiments, interneurons were injected with neurobiotin (Vector Laboratories). The preparations were then fixed in $4 \%$ paraphormaldehyde and $0.1 \%$ glutaraldehyde in PBS and incubated $12 \mathrm{hr}$ in Texas Red-labeled avidin (Vector Laboratories) to visualize neurobiotin-filled interneurons. The preparations were then processed for immunocytochemical reaction as described above. By switching filters in the fluorescence microscope or laser scanning confocal microscope for Texas Red and fluorescein, interneurons were identified as GABAimmunoreactive. Texas Red was not visible with the fluorescein filters, and fluorescein was not visible with the Texas Red filters, thus providing a clear comparison during filter switching.

\section{RESULTS}

\section{Cerebral A interneuron is necessary and sufficient for afterdischarge generation}

A bilaterally symmetrical Cr-Aint neuron was found to be necessary and sufficient for generating afterdischarges in the $\mathrm{Cr}-\mathrm{A}$ neuron network. All neurons in the network were normally silent, with membrane potentials of $\mathrm{Cr}$-A motoneurons varying between -60 and $-66 \mathrm{mV}$ and $\mathrm{Cr}$-Aint neurons varying between -58 and $-63 \mathrm{mV}$. The level of excitability of the Cr-A neuron network, including its ability to generate afterdischarges, significantly varied in different preparations, which apparently reflected the level of feeding arousal (Norekian, 1993). For the first group of experiments, preparations with a high level of excitability were chosen in which each induced $\mathrm{Cr}$-A neuron burst of spikes always triggered afterdischarges. Synchronous discharges were always produced in a simultaneously recorded Cr-Aint neuron (Fig. 1A). When the Cr-Aint neuron was removed from the network activity by injecting hyperpolarizing current, afterdischarges disappeared ( $n=5$ preparations with $5-10$ presentations in each) (Fig. 1A). After release of the Cr-Aint neuron from hyperpolarization, induced $\mathrm{Cr}-\mathrm{A}$ neuron bursts of spikes again triggered discharges in the Cr-Aint neuron and prolonged afterdischarges in the $\mathrm{Cr}-\mathrm{A}$ neuron. In addition, when Cr-Aint neurons were mechanically removed from the ganglia, afterdischarges in the $\mathrm{Cr}-\mathrm{A}$ neuron network disappeared and were never observed in these preparations $(n=3)$.

For the second group of experiments, preparations with a low level of Cr-A neuron network excitability were chosen in which induced $\mathrm{Cr}$-A neuron bursts of spikes did not trigger afterdischarges $($ Fig. $1 B$ ). In all of these preparations, only subthreshold depolarizing potentials were recorded in the Cr-Aint neuron, produced by $\mathrm{Cr}-\mathrm{A}$ neuron bursts of spikes via electrical connections. When the Cr-Aint neuron was depolarized close to the spiking threshold and Cr-A neuron bursts became efficient in 

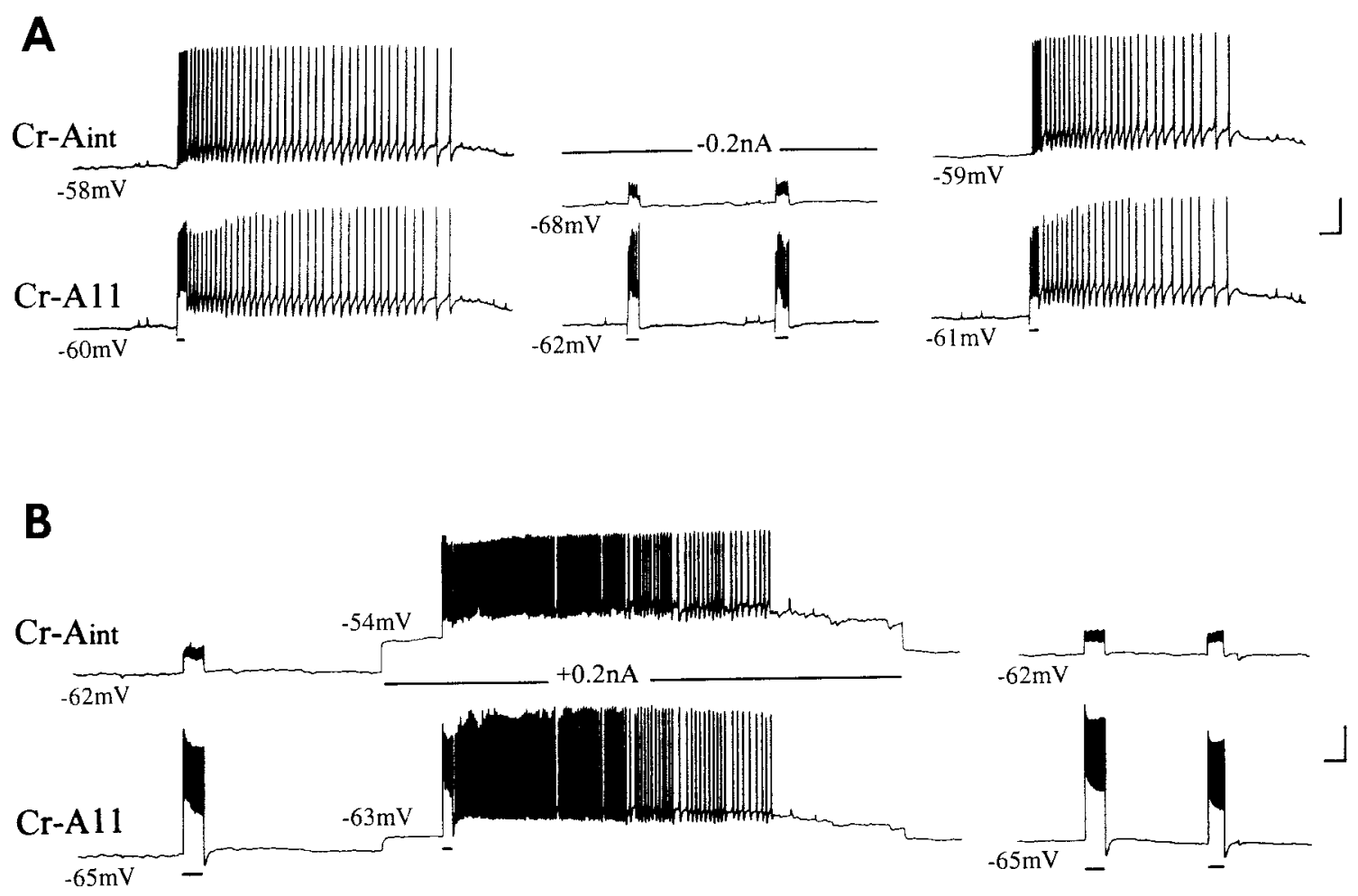

Figure 1. A, Each induced Cr-A11 neuron burst of spikes triggered afterdischarge in this preparation. The Cr-Aint neuron, being activated via an electrical connection, always generated synchronous discharges. When the Cr-Aint neuron was hyperpolarized and did not fire in response to the Cr-A11 neuron burst of spikes, afterdischarges disappeared. $B$, In this preparation, induced Cr-A11 neuron bursts of spikes did not trigger afterdischarges. Note that the Cr-Aint neuron did not fire; only subthreshold depolarization occurred, produced via electrical connection. When the Cr-Aint neuron was depolarized close to the spiking threshold and generated spike activity in response to the Cr-A11 neuron burst of spikes, afterdischarge appeared in the Cr-A11 neuron. Injected currents are indicated by a line below or above the recording trace. Calibration: $10 \mathrm{mV}, 1 \mathrm{sec}$.

triggering $\mathrm{Cr}$-Aint neuron firing, afterdischarges appeared in Cr-A neurons $(n=9$ preparations with $5-10$ presentations in each) (Fig. 1B). All simultaneous recordings of $\mathrm{Cr}-\mathrm{A}$ and $\mathrm{Cr}$-Aint neuron activities demonstrated that afterdischarges appeared only if the Cr-Aint neuron was spiking and were never observed when the Cr-Aint neuron did not produce action potentials $(n=$ 32 preparations). Thus, the Cr-Aint neuron appears to be a key element for afterdischarge generation in the $\mathrm{Cr}-\mathrm{A}$ neuron network.

\section{Synaptic connections between cerebral A interneurons and A motoneurons}

When a single Cr-A neuron was stimulated to trigger afterdischarge activity, it activated the Cr-Aint neuron via electrotonic transmission. Electrical coupling between a Cr-Aint neuron and $\mathrm{Cr}-\mathrm{A}$ motoneurons was nonrectifying and relatively weak (considering high thresholds for spike generation in normal conditions). Coupling coefficients ranged from 0.1 to $0.25(n=7$; measured at resting membrane potentials). These measurements explain why even strong bursts of spikes induced in a $\mathrm{Cr}$-A neuron sometimes were not capable of producing afterdischarges; they could not activate Cr-Aint neurons via electrotonic transmission. On the other hand, induced Cr-Aint neuron bursts always generated $\mathrm{Cr}$-Aint neuron afterdischarges and always produced simultaneous discharges in Cr-A neurons ( $n=32$ preparations, several presentations in each) (Fig. $2 A$ ). The important characteristic of the discharges in both $\mathrm{Cr}$-Aint and $\mathrm{Cr}$-A neurons was a prominent underlying depolarization. These facts suggested the existence of additional excitatory synaptic transmission from Cr-Aint to Cr-A neurons.
In high divalent solution, the Cr-Aint neuron bursts of spikes produced, in addition to the fast electrical membrane changes, slow depolarizations in the Cr-A neurons ( $n=28$ preparations) (Fig. 2B). These slow depolarizations had amplitudes of $7-15 \mathrm{mV}$ and lasted 1-2 sec, depending on the strength of a Cr-Aint neuron burst of spikes. Each spike in a $\mathrm{Cr}$-Aint neuron produced a single EPSP in a Cr-A neuron (Fig. $3 A$ ). This stable one spike/one EPSP ratio persisted in high- $\mathrm{Mg}^{2+} /$ high- $\mathrm{Ca}^{2+}$ saline, suggesting monosynaptic connections ( $n=12$ preparations). Each EPSP had an amplitude between 2 and $6 \mathrm{mV}$ and had a slow decline lasting $\sim 0.5 \mathrm{sec}$. All tested $\mathrm{Cr}-\mathrm{A}$ neurons received those EPSPs from the Cr-Aint neurons, including cells on the dorsal and ventral sides and from the ipsilateral and contralateral cerebral ganglia $(n=18$ different Cr-A neurons in five preparations) (Fig. $3 A, B$ ).

The Cr-Aint neuron is a bilaterally symmetrical cell (Arshavsky et al., 1993; Norekian and Satterlie, 1993a). Contralateral Cr-Aint neurons produced strong excitatory inputs to each other $(n=8)$ (Fig. 4A). Interneurons were found to be electrically coupled, with coupling coefficients between 0.1 and $0.2(n=5)$ (Fig. 4B). In addition to electrical transmission, each Cr-Aint neuron received from its contralateral compatriot slow depolarizing inputs similar to those produced in $\mathrm{Cr}-\mathrm{A}$ neurons. These slow depolarizing potentials persisted in high $-\mathrm{Mg}^{2+} / \mathrm{high}-\mathrm{Ca}^{2+}$ saline, suggesting monosynaptic connections $(n=6)$ (Fig. 4C). Thus, each Cr-Aint neuron could activate its contralateral compatriot and, in turn, receive recurrent excitatory inputs from it. Positive feedback organized via these excitatory reciprocal connections apparently participates in the afterdischarge generation in $\mathrm{Cr}$-Aint neurons and subsequently $\mathrm{Cr}$ - $\mathrm{A}$ neurons. However, it 

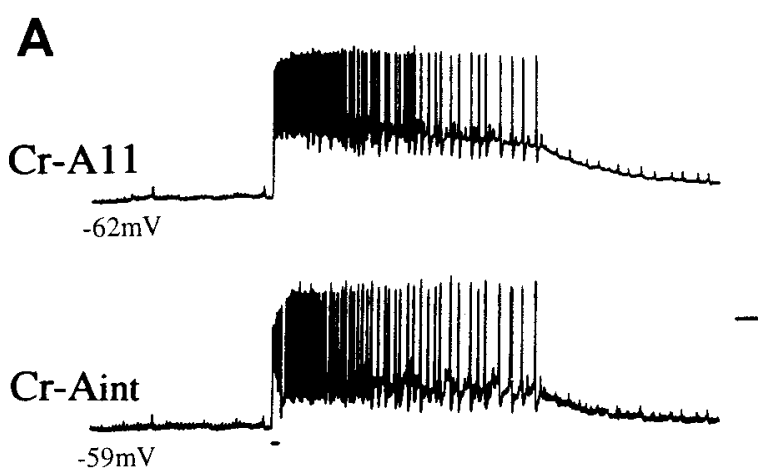

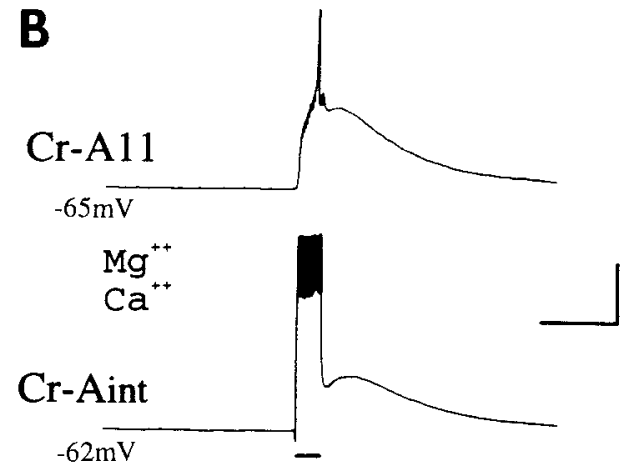

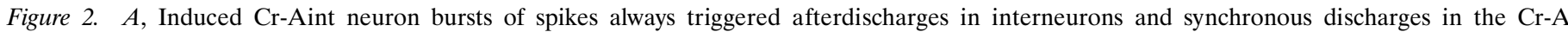

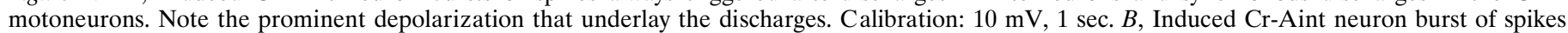

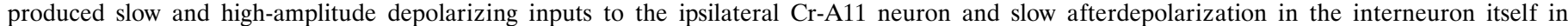
high- $\mathrm{Mg}^{2+} /$ high- $\mathrm{Ca}^{2+}$ solution. Injected currents are indicated by a line below the recording trace. Calibration: $10 \mathrm{mV}$, $0.5 \mathrm{sec}$.

Figure 3. $A$, Each single Cr-Aint neuron spike produced an individual EPSP in a Cr-A neuron and afterdepolarizing potential in the Cr-Aint neuron itself (arrow), which both persisted in high- $\mathrm{Mg}^{2+}$ / high-Ca ${ }^{2+}$ solution. Note that when a depolarizing pulse did not trigger spikes in the Cr-Aint neuron, membrane potential abruptly returned to normal. $B$, The Cr-Aint neuron produced EPSPs in all Cr-A neurons, including those on the opposite side of the cerebral ganglia, such as the $\mathrm{Cr}-\mathrm{A} 2$ neuron. There were two spikes triggered in the Cr-Aint neuron, and two EPSPs recorded in the $\mathrm{Cr}-\mathrm{A} 2$ motoneuron. Afterdepolarization always persisted in a $\mathrm{Cr}$-Aint neuron (arrow). $C$, The $\mathrm{Cr}$ Aint neuron spike was triggered by a short depolarizing impulse of $20 \mathrm{msec}$ duration and $+7 \mathrm{nA}$ amplitude. The arrow indicates the afterdepolarizing potential. Calibration: $A, B, 10 \mathrm{mV}, 0.5 \mathrm{sec}$; $C, 3 \mathrm{mV}, 0.2 \mathrm{sec}$.

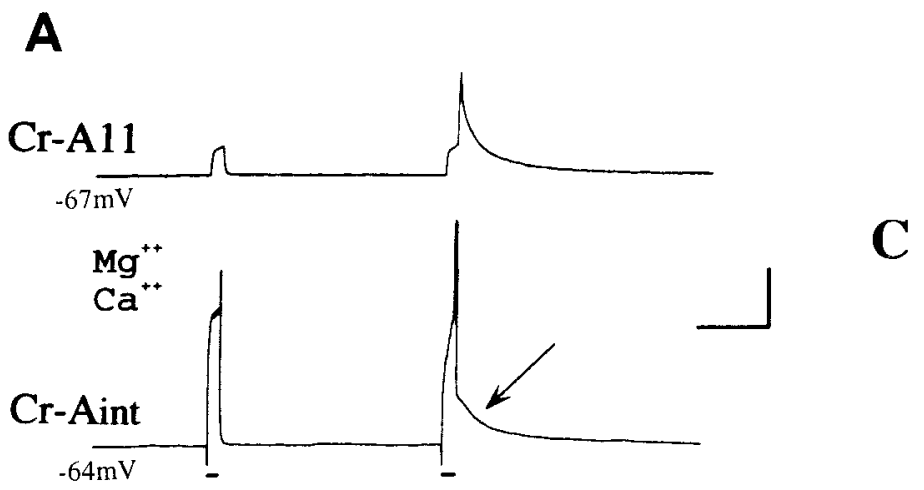

B


was not the only positive feedback, as the following experiments demonstrated.

\section{Afterdepolarizing potentials in A interneurons}

In high- $\mathrm{Mg}^{2+} /$ high-Ca ${ }^{2+}$ saline, induced bursts of spikes in a $\mathrm{Cr}$-Aint neuron produced prominent afterdepolarizations in the stimulated interneuron. Afterdepolarizations were very similar in shape to slow EPSPs in Cr-A neurons, had amplitudes of 7-15 $\mathrm{mV}$, and lasted 1-2 sec, depending on the strength of the Cr-Aint neuron burst $(n=32$ ) (Fig. $2 B$ ). Subthreshold depolarizations never induced $\mathrm{Cr}$-Aint neuron afterdepolarizations; only spikes were effective (Fig. $3 A$ ). Each spike in a $\mathrm{Cr}$-Aint neuron produced an afterdepolarizing potential that had an amplitude between 1 and $4 \mathrm{mV}$ and had a slow decline lasting $\sim 0.5 \mathrm{sec}(n=12)$ (Fig. $3 A, C)$. The appearance of afterdepolarizing potentials after individual spikes in Cr-Aint neurons persisted in high- $\mathrm{Mg}^{2+} /$ high$\mathrm{Ca}^{2+}$ saline ( $n=16$ preparations, several presentations in each) (Fig. 3A,C). Such stable occurrence of afterdepolarizations in high divalent solution suggested that these afterdepolarizations represented a property of the Cr-Aint neuron rather than polysynaptic recurrent inputs. Positive feedback provided by excitatory synaptic connections between contralateral Cr-Aint neurons was certainly not involved, because Cr-Aint neuron afterdepolarizations still persisted when the contralateral neuron was not spiking in high- $\mathrm{Mg}^{2+} /$ high- $\mathrm{Ca}^{2+}$ saline (Fig. 4C). In addition, when one $\mathrm{Cr}$-Aint neuron was mechanically removed from the 



Figure 4. Connections between contralateral Cr-Aint neurons. $A$, Induced burst of spikes in the right Cr-Aint neuron produced a prominent spike activity in the left Cr-Aint neuron. Calibration: $10 \mathrm{mV}, 1 \mathrm{sec} . B$, Left and right Cr-Aint neurons were electrically coupled. Electrical coupling was demonstrated in high- $\mathrm{Mg}^{2+}$ solution by applying negative or positive current pulses to one cell and recording similar but attenuated responses in the other cell at the same time. Calibration: $10 \mathrm{mV}, 1 \mathrm{sec}$. $C$, Left and right Cr-Aint neurons produced slow depolarizing potentials in each other, which persisted in high- $\mathrm{Mg}^{2+} /$ high-Ca ${ }^{2+}$ solution. Note that slow afterdepolarization persisted in the right $\mathrm{Cr}$-Aint neuron, although no spikes were fired in the left Cr-Aint neuron. Injected currents are indicated by a line below the recording trace. Calibration: $10 \mathrm{mV}, 0.5 \mathrm{sec}$.

ganglia (cell body with extended part of an axon and secondary branches), afterdischarges still persisted in the network $(n=5)$.

Afterdepolarizing potentials triggered by each single Cr-Aint neuron spike could have two theoretically possible mechanisms. First, spikes could activate slow voltage-sensitive inward current, which would depolarize the Cr-Aint neuron membrane potential. Slow inward currents play a major role in the generation of endogenous bursting activity in different pacemaker neurons (Thompson and Smith, 1976; Adams et al., 1980; Bulloch and Willows, 1981). Second, afterdepolarizations could be a result of the synaptic self-excitatory inputs. The fact that Cr-Aint neuron afterdepolarizations were very similar in shape to the simultaneously induced Cr-A neuron EPSPs suggested that they could be excitatory synaptic inputs produced by interneurons to themselves. Moreover, repetitive Cr-Aint neuron stimulation produced a prominent decrement of afterdepolarizations, with the same degree and time course as the parallel decrement of $\mathrm{Cr}-\mathrm{A}$ neuron EPSPs $(n=12)$. One general characteristic of any synaptic transmission is that it is a $\mathrm{Ca}^{2+}$-dependent process. The goal of the first group of experiments was to test whether the Cr-Aint neuron afterdepolarizations were $\mathrm{Ca}^{2+}$-dependent. When normal seawater in the recording dish was replaced with zero- $\mathrm{Ca}^{2+}$ seawater, Cr-Aint neuron afterdepolarizations disap- peared, along with slow EPSPs in Cr-A motoneurons $(n=4)$ (Fig. $5 A)$. Cr-Aint neuron afterdepolarizations and $\mathrm{Cr}-\mathrm{A}$ neuron $\mathrm{EP}$ SPs were also completely and reversibly blocked by $\mathrm{Ca}^{2+}$ channel antagonists: $120 \mathrm{~mm} \mathrm{Mg}{ }^{2+}(n=6)$ (Fig. $\left.5 B\right)$ and $0.2 \mathrm{mM} \mathrm{Cd}^{2+}$ $(n=3)$. In addition, the Cr-Aint neuron afterdepolarizations, as well as $\mathrm{Cr}-\mathrm{A}$ neuron EPSPs, were enhanced in high-Ca ${ }^{2+}$ saline (30 mm; $n=3$ ). These experiments concluded that $\mathrm{Cr}$-Aint neuron afterdepolarizing potentials were $\mathrm{Ca}^{2+}$-dependent and that they could be synaptic inputs. However, there are too many $\mathrm{Ca}^{2+}$-dependent processes in neural networks, and the only way to unequivocally establish synaptic transmission as a mechanism of Cr-Aint neuron afterdepolarizations would be to demonstrate the effects of the specific pharmacological agents.

\section{Depolarizations produced by $A$ interneurons are GABAergic excitatory synaptic inputs}

In addition to $\mathrm{Cr}-\mathrm{A}$ motoneurons, $\mathrm{Cr}$-Aint neurons also targeted buccal cone retractor $\mathrm{Cr}-\mathrm{B}$ motoneurons (Norekian and Satterlie, 1993a). Cr-Aint neurons produced strong inhibitory inputs to Cr-B neurons, which temporally interrupted their spontaneous spike activities $(n=5)$ (Fig. 6). These inhibitory hyperpolarizing inputs persisted in high- $\mathrm{Mg}^{2+} /$ high- $\mathrm{Ca}^{2+}$ seawater, suggesting direct connection between neurons. The only neurotransmitter, 
A

Figure 5. Afterdepolarizations in the Cr-Aint neurons were $\mathrm{Ca}^{2+}$-dependent. $A$, Cr-Aint neuron afterdepolarizations and slow EPSPs in the Cr-A motoneurons disappeared in zero- $\mathrm{Ca}^{2+}$ seawater. The remaining excitatory input to the Cr-A11 neuron from the Cr-Aint neuron was carried via electrical coupling. $B$, High- $\mathrm{Mg}^{2+}$ saline completely and reversibly blocked afterdepolarizations in the Cr-Aint neurons and slow EPSPs in the Cr-A motoneurons. All controls were conducted in the seawater with the increased concentration of $\mathrm{Mg}^{2+}$ and $\mathrm{Ca}^{2+}(1.75 \times$ normal or half of the high- $\mathrm{Mg}^{2+} /$ high-Ca ${ }^{2+}$ solution) to prevent prolonged firing and to unmask slow depolarizing responses in neurons. Calibration: $10 \mathrm{mV}, 1 \mathrm{sec}$.


Figure 6. Induced bursts of spikes in the Cr-Aint neuron produced strong inhibitory inputs to the buccal cone retractor motoneurons, the Cr-B motoneurons. IPSPs in the Cr-B motoneurons persisted in high$\mathrm{Mg}^{2+} /$ high-Ca ${ }^{2+}$ seawater. Calibration: 15 $\mathrm{mV}, 2$ sec.
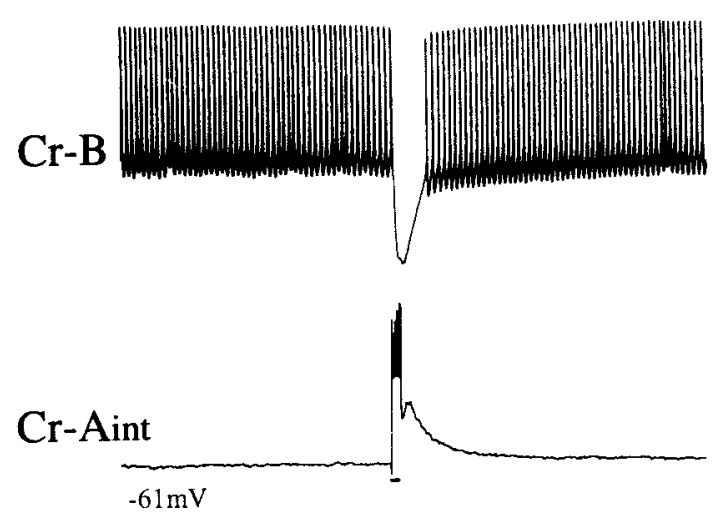

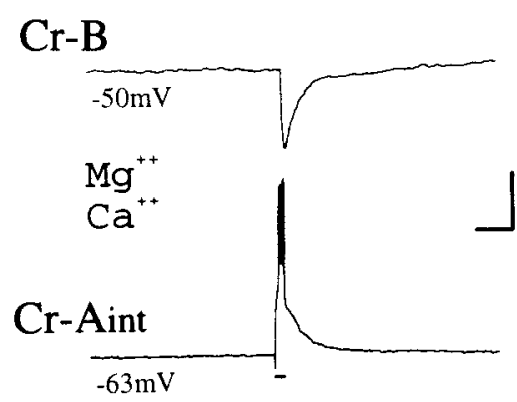

which mimicked all effects produced by $\mathrm{Cr}$-Aint neurons, was GABA. GABA has been shown previously to exert a strong excitatory effect on Cr-A neurons (Arshavsky et al., 1993; Norekian and Satterlie, 1993b). In high- $\mathrm{Mg}^{2+}$ seawater, $10 \mu \mathrm{M}$ GABA produced prominent depolarization of $\mathrm{Cr}-\mathrm{A}$ motoneurons and hyperpolarization of Cr-B motoneurons $(n=4)$ (Fig. 7) (Norekian and Satterlie, 1993b). Exogenous $10 \mu \mathrm{M}$ GABA also produced a prominent depolarization of a $\mathrm{Cr}$-Aint neuron itself $(n=5)$ (Fig. 7). Thus, GABA mimicked the effects induced by a $\mathrm{Cr}$-Aint neuron and was suggested as its possible neurotransmitter.

The amplitude of $10 \mu \mathrm{M}$ GABA-induced depolarizations in the $\mathrm{Cr}$-A and Cr-Aint neurons was $\sim 20 \mathrm{mV}$ when measured from the resting membrane potentials $(n=12)$. When $\mathrm{Cr}-\mathrm{A}$ motoneuron or Cr-Aint neuron membrane potentials were depolarized to +20 $\mathrm{mV}$ by injecting a positive current via a second electrode, GABAinduced depolarizations decreased in amplitude to $5 \mathrm{mV}$ but still did not reverse $(n=5)$. Similarly, Cr-Aint neuron-induced EPSPs in the $\mathrm{Cr}-\mathrm{A}$ motoneurons did not reach a reversal potential when the motoneuron membrane potential was depolarized to $+20 \mathrm{mV}$ $(n=7)$. GABA-induced depolarizations, as well as $\mathrm{Cr}-\mathrm{A}$ motoneuron EPSPs and Cr-Aint neuron afterdepolarizations, were associated with increases in membrane conductance $(n=10)$. When seawater in the recording chamber was replaced with $2 \%$ $\mathrm{Na}^{+}$solution, GABA-induced depolarizations in the Cr-A and Cr-Aint neurons completely disappeared (GABA concentration of $50 \mu \mathrm{M} ; n=5$ ) (Fig. 8). After a 5 min wash in seawater, GABA again produced prominent depolarizing responses. Replacement of seawater with zero $\mathrm{Ca}^{2+}$ and $1 \mathrm{mM} \mathrm{Co}^{2+}$ solution did not influence GABA-induced depolarizations (50 $\mu \mathrm{M} \mathrm{GABA;} n=3$ ). These observations suggest that GABA-induced depolarizations are $\mathrm{Na}^{+}$-dependent.

The GABA antagonists bicuculline, phaclofen, saclofen, and picrotoxin, in concentrations of $50 \mu \mathrm{M}$ and $1 \mathrm{mM}$, did not block 1 $\mu \mathrm{M}$ GABA-induced responses $(n=9)$. They also did not block Cr-A motoneuron EPSPs and Cr-Aint neuron afterdepolarizations $(n=12)$. The GABA antagonist 5 -aminovaleric acid (50 




Figure 7. Effects of $10 \mu \mathrm{M}$ GABA on neurons Cr-B1, Cr-A1, and Cr-Aint in high- $\mathrm{Mg}^{2+}$ solution. Note that GABA hyperpolarized the Cr-B1 neuron and depolarized the Cr-A1 motoneuron and the Cr-Aint neuron. Calibration: $10 \mathrm{mV}, 1 \mathrm{sec}$.
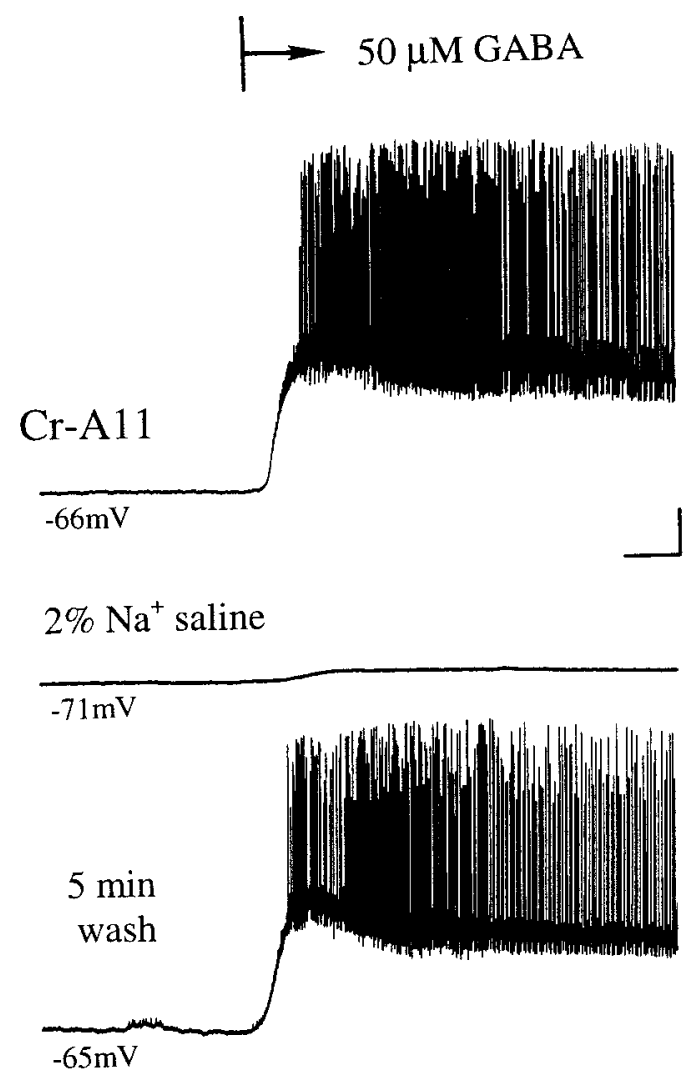

Figure 8. GABA-induced depolarization and activation of the Cr-A neurons disappeared in $2 \% \mathrm{Na}^{+}$saline. The effect was reversible, and the GABA response was restored immediately after wash in normal seawater. Calibration: $10 \mathrm{mV}, 3 \mathrm{sec}$.

$\mu \mathrm{M})$ produced a prominent depolarization and activation of the $\mathrm{Cr}-\mathrm{A}$ and Cr-Aint neurons $(n=4)$. Ten micromolar 5-aminovaleric acid depolarized neuron membrane potential 5-9 $\mathrm{mV}$ above the resting potential without initiating spikes and demonstrated a reversible blocking effect on the $1 \mu \mathrm{M}$ GABA-

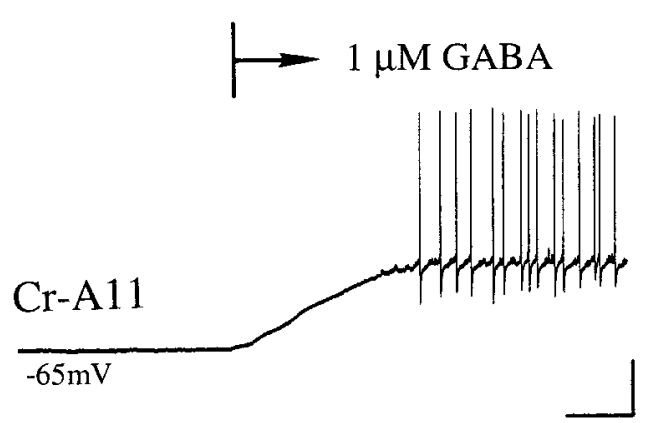

\section{$50 \mu \mathrm{M}$ piperidine- $4 \mathrm{~S}$}

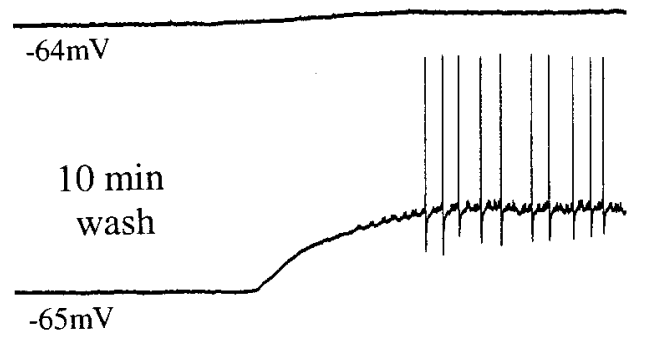

Figure 9. The GABA antagonist piperidine-4-S completely and reversibly blocked GABA-induced depolarization of the Cr-A neurons. Calibration: $10 \mathrm{mV}, 3 \mathrm{sec}$.

induced responses $(n=3)$. It also reversibly blocked chemical transmission from a Cr-Aint neuron to $\mathrm{Cr}$-A motoneurons and Cr-Aint neuron afterdepolarizations $(n=7)$. However, the best results were obtained with piperidine-4-S, which acts as a GABA antagonist in some systems (Woodward et al., 1993). Fifty micromolar piperidine-4-S did not significantly alter $\mathrm{Cr}$-A and $\mathrm{Cr}$-Aint neuron membrane potentials. Only a slight depolarization of $<2$ $\mathrm{mV}$ amplitude was observed in some experiments. Responses of Cr-A and Cr-Aint neurons to $1 \mu \mathrm{M}$ GABA were completely and reversibly blocked by $50 \mu \mathrm{M}$ piperidine-4-S, indicating that it works as a GABA antagonist in this system $(n=3)$ (Fig. 9). Fifty micromolar piperidine-4-S reversibly blocked chemical transmission from Cr-Aint neurons to Cr-A motoneurons $(n=8)$ (Fig. $10 A$ ). In these experiments, slow EPSPs in the Cr-A motoneurons completely disappeared, and the remaining fast responses were very similar in amplitude and shape to the $\mathrm{Cr}$-A neuron responses in zero- $\mathrm{Ca}^{2+}$ or high- $\mathrm{Mg}^{2+}$ experiments (Fig. 5). Apparently, only fast electrical transmission remained functional between neurons. At the same time, $50 \mu \mathrm{M}$ piperidine-4-S completely and reversibly blocked afterdepolarizations in the $\mathrm{Cr}$-Aint neurons $(n=8)$ (Fig. 10A). Nipecotic acid, a GABA uptake inhibitor, significantly enhanced both slow Cr-A neuron EPSPs and Cr-Aint neuron afterdepolarizations, increasing their amplitudes but most dramatically their durations $(n=8)$ (Fig. 10B). Fifty micromolar nipecotic acid produced a twofold to threefold increase in the duration of both Cr-A neuron EPSPs and Cr-Aint neuron afterdepolarizations compared with controls. Application of the nipecotic acid always resulted in the $3-5 \mathrm{mV}$ depolarization of $\mathrm{Cr}-\mathrm{A}$ and $\mathrm{Cr}$-Aint neurons and required the adjustment of the stimulating current to keep the firing frequency of the induced bursts of spikes in the Cr-Aint neurons constant. These experiments demonstrated that synaptic transmission from interneurons to Cr-A motoneurons was GABAergic. They also provided evidence that afterdepolarizations in $\mathrm{Cr}$-Aint neurons are recurrent synaptic inputs, with GABA being a neurotransmitter. 

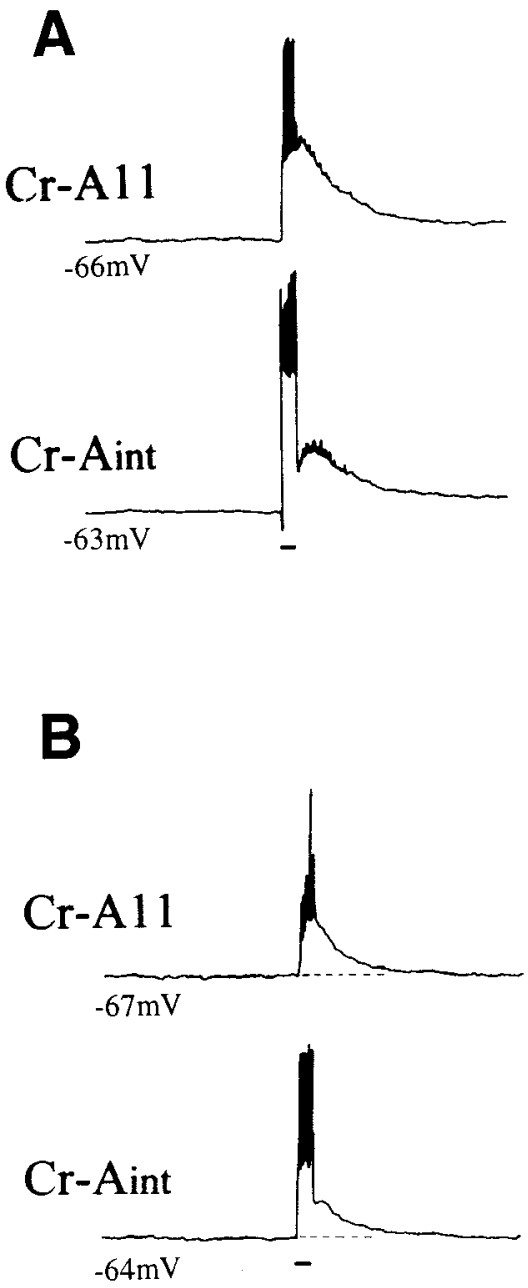



Figure 10. A, The GABA antagonist piperidine-4-S completely and reversibly blocked Cr-Aint neuron afterdepolarizations and slow EPSPs in the Cr-A11 neuron. Apparently, only fast electrical transmission remained functional. $B$, Nipecotic acid, a GABA uptake inhibitor, increased the duration of both Cr-Aint neuron afterdepolarizations and slow EPSPs in the Cr-A11 neuron. All experiments were conducted in the seawater with the increased concentration of $\mathrm{Mg}^{2+}$ and $\mathrm{Ca}^{2+}\left(1.75 \times\right.$ normal or half of the high- $\mathrm{Mg}^{2+} /$ high- $\mathrm{Ca}^{2+}$ solution) to prevent prolonged firing and to unmask slow depolarizing responses in neurons. Calibration: $10 \mathrm{mV}, 1 \mathrm{sec}$.

\section{GABA immunocytochemistry and double-labeling experiments}

Because synaptic transmission from a Cr-Aint neuron to its target cells was sensitive to GABA pharmacology, Cr-Aint neurons were expected to contain GABA. Validation of this expectation was important, because it would provide additional confirmation of its GABAergic nature and the direct nature of the synapses studied. One technique, which could demonstrate that Cr-Aint neurons contained GABA, was immunocytochemistry. GABA immunoreactivity was studied in 16 whole-mount preparations. General distribution of GABA-immunoreactive neurons in the CNS was similar to that described by Arshavsky et al. (1993). There were small groups of immunoreactive neurons in the buccal, pedal, and cerebral ganglia, and no stained neurons in the pleural and intestinal ganglia.

Immunoreactive cells in the buccal ganglia included three pairs of small neurons, which sent their axons into the cerebrobuccal connectives (Fig. 11). The pedal ganglia contained a cluster of five to six small neurons in the lateral region, one cell in the anterior region on the dorsal surface, and two cells in the medial region on

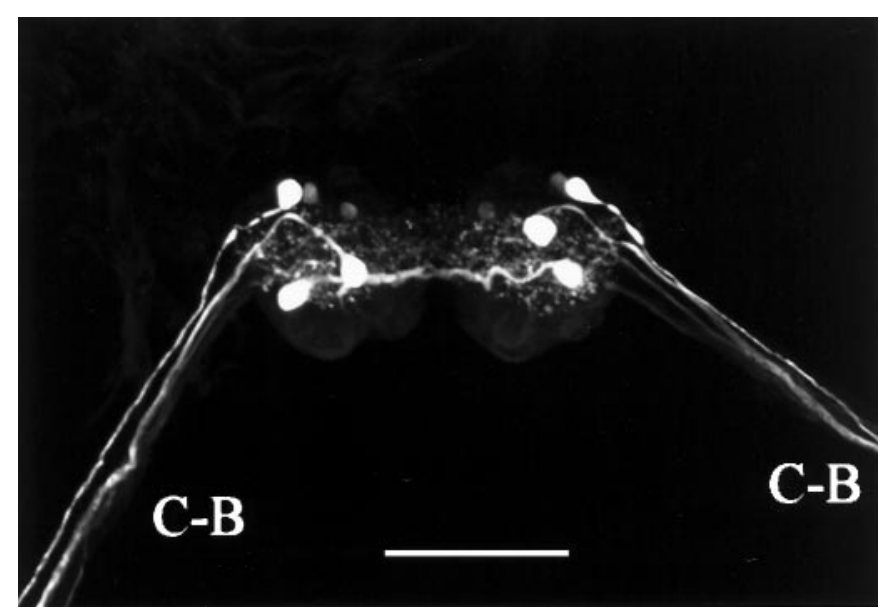

Figure 11. GABA immunoreactivity in the buccal ganglia: a composite confocal microscope reconstruction from several optical sections. Note the stained processes exiting the ganglia into the cerebrobuccal connectives $(C-B)$. Scale bar, $100 \mu \mathrm{m}$. 

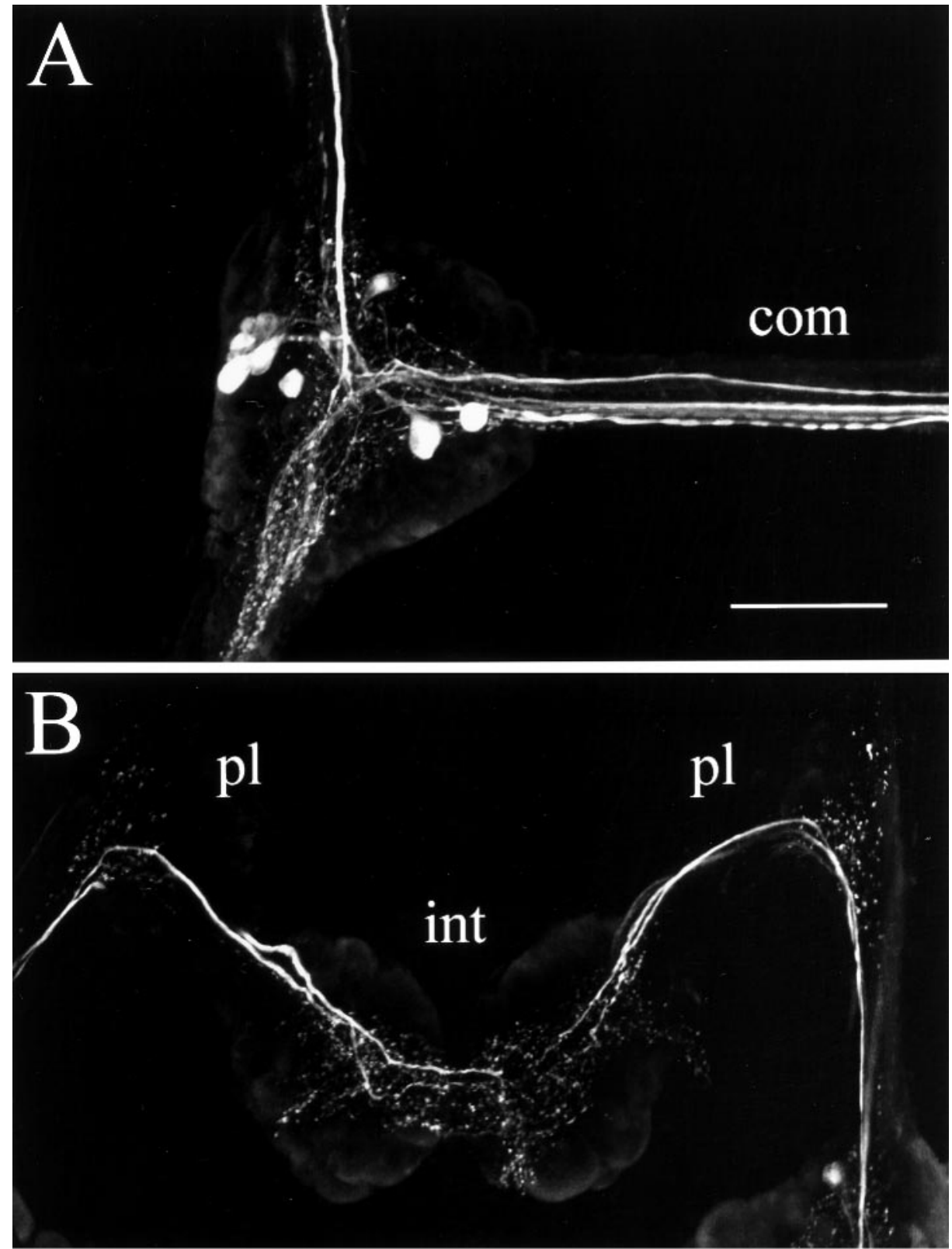

Figure 12. GABA immunoreactivity in the central ganglia. $A$, Left pedal ganglion. Note several stained processes in the pedal commissure (com). B, Pleural ( $p l)$ and intestinal (int) ganglia. Note that there are no stained cell bodies in these ganglia. All images represent a composite confocal microscope reconstruction. Scale bar, $200 \mu \mathrm{m}$. the ventral surface (Fig. 12A). Although the pleural and intestinal ganglia did not contain any immunoreactive cell bodies, their neuropiles were innervated by immunoreactive processes (Fig. $12 \mathrm{~B}$ ). It appeared that only two symmetrical pairs of immunoreactive axon branches innervated the neuropile of the intestinal ganglia, and two pairs innervated the neuropile of the pleural ganglia. Approximately 10 pairs of immunoreactive neurons were found in the cerebral ganglia (Fig. 13B). One small brightly stained bilaterally symmetrical neuron was located near the head nerves. A group of small neurons was spread in the center of the dorsal surface of each cerebral ganglion. One bilaterally symmetrical neuron of middle size showed strong immunoreactivity and had a location similar to that of a Cr-Aint neuron, suggesting that it might be the same cell (Fig. 13B).

Double-labeling experiments undoubtedly demonstrated that the Cr-Aint neuron is GABA-immunoreactive $(n=10)$. One $\mathrm{Cr}$-Aint neuron in each preparation, from the left or right cere- bral ganglion, was injected with neurobiotin and visualized with Texas Red (Fig. 13A). The same preparation was then processed for GABA immunoreactivity with the fluorescein-labeled secondary antibody (Fig. 13B). By switching filters in the same preparation, the Cr-Aint neuron was identified as a GABAimmunoreactive neuron. Texas Red and fluorescein had very distinct emission wavelengths and were visible only in their own set of filters, thus allowing complete separation of images and clear interpretation of data.

One unusual observation, which resulted from the doublelabeling experiments, was the lack of dye coupling in this system of electrically coupled neurons after neurobiotin injections (Fig. $13 A$ ). Neurobiotin is known to produce dye coupling, even when other junction-permeable dyes, such as Lucifer yellow, are not efficient (Vaney, 1991). The lack of neurobiotin coupling in the Cr-A neuron network could be explained by the specificity of the gap junctions, such as narrow junctional channels, and/or by the 


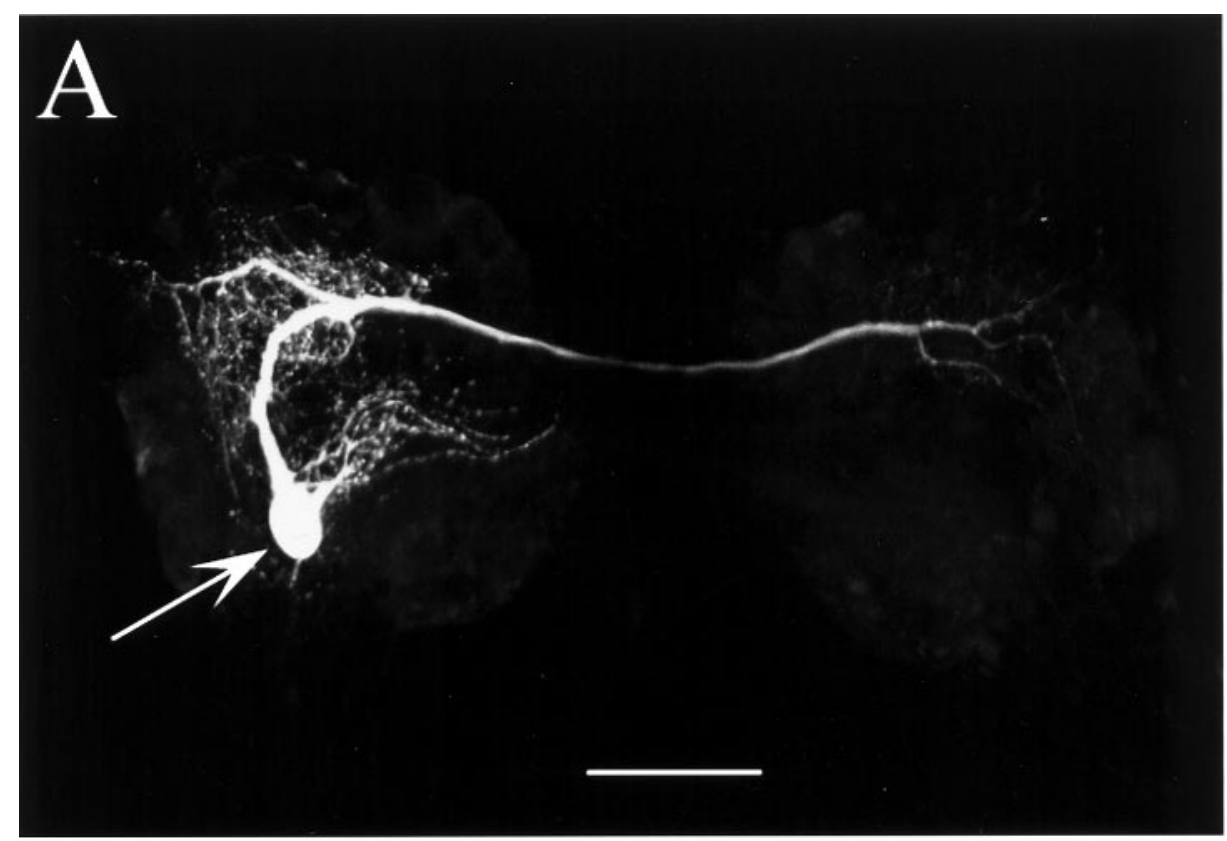

Figure 13. Double-labeling experiment. $A$, Image of the cerebral ganglia obtained via Texas Red filters. A single Cr-Aint neuron was filled with neurobiotin and visualized by Texas Red. The cell body is indicated by an arrow. $B$, Image of the same cerebral ganglia obtained via fluorescein filters. This image shows GABA immunoreactivity in the cerebral ganglia (GABA antisera were labeled with fluorescein). The cell body of a Cr-Aint neuron is indicated by an arrow. All images represent a composite confocal microscope reconstruction. Scale bars, $200 \mu \mathrm{m}$.



lack of a prolonged incubation period before fixation, which was always under $30 \mathrm{~min}$.

\section{DISCUSSION}

\section{Mechanisms of afterdischarge generation in the prey capture network}

Afterdischarge activity in the Clione prey capture network has an important functional implication for the feeding behavior. It is responsible for transformation of a brief sensory input from a prey into a long-lasting motor output-prolonged protraction of the prey capture appendages (Norekian, 1993). The focus of the current investigation is how these afterdischarges are generated. It has been demonstrated previously that afterdischarge activity is not attributable to intrinsic properties of individual Cr-A motoneurons (Norekian, 1993). The present research revealed that Cr-Aint interneurons and their synaptic connections are necessary for afterdischarge generation.
The emerging picture of synaptic connections between Cr-Aint neurons and Cr-A motoneurons is shown in Figure 14. Cr-Aint neurons and $\mathrm{Cr}-\mathrm{A}$ motoneurons are electrically coupled. There is also a slow excitatory synaptic transmission from $\mathrm{Cr}$-Aint neurons to $\mathrm{Cr}-\mathrm{A}$ motoneurons. The excitatory synaptic inputs produced by Cr-Aint neurons were found to be GABAergic, as demonstrated by the use of GABA antagonists, uptake inhibitors, and doublelabeling experiments, which showed that Cr-Aint neurons are GABA-immunoreactive. GABA was originally known as an inhibitory neurotransmitter. However, GABA-induced depolarizing effects were found in several neural systems (Gallagher et al., 1978; Ogata, 1987; Pfeiffer-Linn and Glantz, 1989; El-Beheiry and Puil, 1990; Mercuri et al., 1991; Michelson and Wong, 1991). In Clione, it appears that GABA works primarily as an excitatory transmitter in the feeding system (Arshavsky et al., 1993; Norekian and Satterlie, 1993b). GABA-induced depolarizations in the $\mathrm{Cr}$-Aint neurons and $\mathrm{Cr}-\mathrm{A}$ motoneurons have very high 


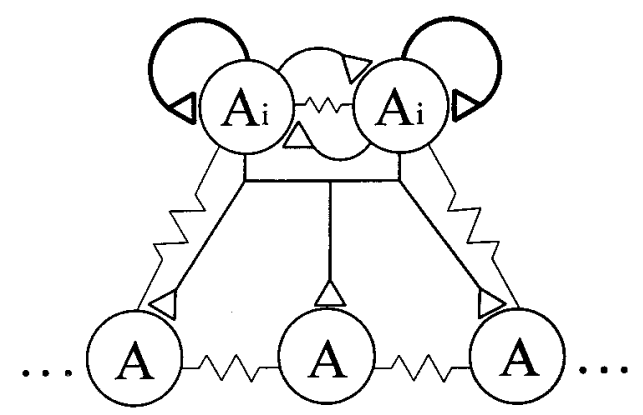

Figure 14. Schematic representation of the synaptic connections in the $\mathrm{Cr}-\mathrm{A}$ neuron network. Each of the two bilaterally symmetrical $\mathrm{Cr}$-Aint neurons $(\mathrm{Ai})$ produces monosynaptic excitatory inputs (open triangles) to itself and to the contralateral interneuron. These connections organize a positive feedback that underlies afterdischarge activity in $\mathrm{Cr}$-Aint neurons. $\mathrm{Cr}$-Aint neurons recruit all other $\mathrm{Cr}$-A neurons in the synchronous firing via excitatory monosynaptic inputs to all of them. In addition to the chemical synapses, all neurons are electrically coupled.

amplitudes, easily reach spike thresholds, and strongly activate the target cells. What makes these responses unusual compared with the previously known GABA-produced depolarizations is their $\mathrm{Na}^{+}$dependence and atypical pharmacology. Further investigation of these apparently novel GABAergic receptors will be an important direction for future experiments.

The Cr-Aint neurons organize three different pathways in the prey capture network, which provide the positive feedback necessary for sustaining prolonged spike activity. The first pathway includes electrical coupling and slow chemical transmission from the $\mathrm{Cr}$-Aint neurons to $\mathrm{Cr}$-A motoneurons. Cr-Aint neurons produce slow excitatory inputs to all $\mathrm{Cr}$ - $\mathrm{A}$ neurons in the network, and Cr-A motoneurons in turn activate the Cr-Aint neurons via electrotonic transmission. Electrical coupling between Cr-Aint neurons and $\mathrm{Cr}$-A motoneurons, which all have high thresholds for spike generation, is relatively weak and does not efficiently propagate the impulses at the resting membrane potentials. However, when slow depolarizing inputs produced by Cr-Aint neurons bring all neurons close to the spiking thresholds, even weak electrical coupling could turn into a very efficient pathway for impulse transmission between neurons. The fact that neuron firing is very synchronized in the network during afterdischarges confirms the active involvement of electrical coupling (Norekian, 1993).

The second positive feedback mechanism, which apparently participates in sustaining neuron firing, is provided by reciprocal connections between contralateral Cr-Aint neurons (Fig. 14). Contralateral Cr-Aint neurons are electrically coupled. In addition, each interneuron produces slow excitatory synaptic inputs to its compatriot, similar to the synaptic inputs in the $\mathrm{Cr}-\mathrm{A}$ motoneurons. Thus, when a brief sensory input induces a short burst of activity in the Cr-Aint neurons, each interneuron produces excitatory inputs to the contralateral interneuron, which returns this excitation. This recurrent excitation via the contralateral interneuron can sustain prolonged firing in the Cr-Aint neurons. $\mathrm{Cr}$-Aint neuron firing in turn activates all $\mathrm{Cr}-\mathrm{A}$ motoneurons in the network via excitatory synaptic transmission, and motoneurons fire as long as Cr-Aint neurons are active.

The third positive feedback mechanism is restricted to each individual Cr-Aint neuron. Single spikes in the Cr-Aint neurons produce afterdepolarizing potentials, which persist in high divalent solution. Induced bursts of spikes in a Cr-Aint neuron pro- duce afterdepolarizations that are prominent enough to initiate the following set of spike activity, thus creating a positive feedback mechanism. Afterdepolarizations are sensitive to GABA pharmacology and apparently represent recurrent GABAergic excitatory inputs. How do Cr-Aint neurons activate themselves? Autoexcitatory effects of released neurotransmitters have been shown in previous studies. One well studied example is the group of bag cells in the mollusc Aplysia (Brown and Mayeri, 1989; Loechner and Kaczmarek, 1994). After stimulation, bag cells produce prolonged afterdischarges that typically last 15-30 min. Positive feedback responsible for this sustained firing activity is organized by autoexcitatory transmission of the several bag cell neuropeptides, which are nonsynaptically released during neuron firing. It appears highly unlikely that $\mathrm{Cr}$-Aint neurons nonsynaptically release GABA. Cr-Aint neurons and $\mathrm{Cr}-\mathrm{A}$ motoneurons do not represent a compact cluster of neurons as the bag cells do; their cell bodies and axons are spread around the entire cerebral ganglia. Nevertheless, they are the only neurons involved in the afterdischarge activity, although many other cerebral neurons are also sensitive to GABA (my unpublished observations). Cr-Aint neurons target only specific neurons, suggesting the existence of the specific synapses. Thus, afterdepolarizing potentials in the Cr-Aint neurons apparently represent GABAergic excitatory autaptic inputs (Fig. 14).

\section{Self-excitatory synapses and afterdischarge generation}

The existence of autapses in the brain has been documented by many morphological studies, starting from the work in rabbit neocortex by Van der Loos and Glaser (1972), who proposed the term autapse to describe a synapse between a neuron and a branch of its own axon. Autaptic connections were later found in various brain regions, such as monkey neostriatum (DiFiglia et al., 1976), cat substantia nigra (Karabelas and Purpura, 1980), rat striatum (Preston et al., 1980), and cat visual cortex (Tamás et al., 1995). Detailed light microscopic observations, combined with electron microscopy study, were recently accomplished in rat neocortex (Lubke et al., 1996) and cat visual cortex (Tamás et al., 1997). Thus, there are substantial morphological data obtained from several brain structures in different species, which demonstrate that autapses may be fairly common in the brain. Surprisingly, a physiological description of autapses and investigation of their possible significance in brain functioning are almost absent. Both excitatory and inhibitory autapses have been reported and extensively studied in cell culture (Crain, 1971; Bekkers and Stevens, 1991; Segal, 1991, 1994; Shi and Rayport, 1994). However, their formation in culture could be argued as an aberration in the absence of appropriate postsynaptic targets. Physiological studies in vivo are limited to only three reports describing inhibitory autapses. The first physiological study, which was performed on the cholinergic buccal neurons of the mollusc Aplysia, identified inhibitory autaptic connections as a mechanism of hyperpolarizing potentials that followed action potentials in these neurons and slowed their firing (Gardner, 1977; White and Gardner, 1981). The second report demonstrated that axon collaterals of the rat neostriatal spiny neurons mediate recurrent inhibition, a portion of which involves autaptic synapses (Park et al., 1980). A third, very recent report described autaptic inhibitory currents in interneurons from rat cerebellar slices and also suggested that their functional role is inhibition of interneuron firing during high-frequency discharges (Pouzat and Marty, 1998). 
The present study suggests the existence of GABAergic excitatory autaptic connections in two Cr-Aint neurons, which are the key elements of the Clione prey capture neural network. Recurrent excitation organized by these autaptic connections apparently serves an important functional role, providing an efficient positive feedback for sustaining spike activity in the Cr-Aint neurons and generating afterdischarges in the entire network. A pair of interneurons [cerebral prey capture interneuron (Cr-PC)] has been previously identified in the cerebral ganglia that also produced electrical and excitatory connections to Cr-A motoneurons but without self-excitatory inputs (Norekian and Satterlie, 1995). These interneurons did not generate afterdischarge activity and triggered discharges in the $\mathrm{Cr}-\mathrm{A}$ neural network only occasionally, when Cr-Aint neurons were apparently activated (Norekian and Satterlie, 1995). This comparison suggests that self-excitatory synapses in a single pair of key interneurons $(\mathrm{Cr}-$ Aint neurons) can influence dramatically the properties of the entire network and are very important for afterdischarge generation.

Although the physiological evidence presented here suggests that afterdepolarizing potentials in the Cr-Aint neurons represent the excitatory autaptic inputs, one alternative hypothesis exists that can explain the same data. According to this hypothesis, Cr-Aint neuron-induced excitatory synaptic inputs could produce depolarization of numerous $\mathrm{Cr}$-A motoneurons, which in turn could feed back via electrical connections to maintain a depolarization in the Cr-Aint neurons. Electrical coupling measured between pairs of interneurons and motoneurons was found to be too weak to have such an effect. However, simultaneous depolarization of $\sim 26$ motoneurons could theoretically provide a substantial convergent current source back to Cr-Aint neurons. Unfortunately, there are no known blockers of electrical coupling in the Clione system, which would allow obtaining a direct physiological solution. One indirect consideration that favors the "excitatory autapses" hypothesis is the comparison with $\mathrm{Cr}-\mathrm{PC}$ interneurons, which also have electrical connections with $\mathrm{Cr}-\mathrm{A}$ motoneurons and produce strong excitatory synaptic inputs to all of them (Norekian and Satterlie, 1995). Although their synaptic transmission produces strong depolarization of all $\mathrm{Cr}-\mathrm{A}$ motoneurons, Cr-PC interneurons do not have afterdepolarizing potentials and do not generate afterdischarge activity. However, the best and the final solution to the dilemma can be provided only by further computational and electron microscopical analysis. What this study unequivocally demonstrated is that a pair of GABAergic Cr-Aint neurons with their excitatory synaptic and electrical connections is responsible for sustaining prolonged discharges in a prey capture neural network of Clione.

\section{REFERENCES}

Adams DJ, Smith SJ, Thompson SH (1980) Ionic currents in molluscan somata. Annu Rev Neurosci 3:141-167.

Arshavsky YI, Deliagina TG, Gamkrelidze GN, Orlovsky GN, Panchin YV, Popova LB, Shupliakov OV (1993) Pharmacologically induced elements of the hunting and feeding behavior in the pteropod mollusk Clione limacina. I. Effects of GABA. J Neurophysiol 69:512-521.

Bekkers JM, Stevens CF (1991) Excitatory and inhibitory autaptic currents in isolated hippocampal neurons maintained in cell culture. Proc Natl Acad Sci USA 88:7834-7838.

Brown RO, Mayeri E (1989) Positive feedback by autoexcitatory neuropeptides in neuroendocrine bag cells of Aplysia. J Neurosci 9:1443-1451.

Bulloch AGM, Willows AOD (1981) Physiological basis of feeding behavior in Tritonia diomedea. III. Role of depolarizing afterpotentials. J Neurobiol 12:515-532.
Crain SM (1971) Intracellular recordings suggesting synaptic functions in chick embryo spinal sensory ganglion cells isolated in vitro. Brain Res 26:188-191.

DiFiglia M, Pasik P, Pasik T (1976) A Golgi study of neuronal types in the neostriatum of monkeys. Brain Res 114:245-256.

Douglas RJ, Koch C, Mahowald M, Martin KAC, Suarez, HH (1995) Recurrent excitation in neocortical circuits. Science 269:981-985.

El-Beheiry H, Puil E (1990) Unusual features of GABA responses in layers IV-V neurons of neocortex. Neurosci Lett 119:83-85.

Gallagher JP, Higashi H, Nishi S (1978) Characterization and ionic basis of GABA-induced depolarizations recorded in vitro from cat primary afferent neurons. J Physiol (Lond) 275:263-282.

Gardner D (1977) Voltage-clamp analysis of a self-inhibitory synaptic potential in the buccal ganglia of Aplysia. J Physiol (Lond) 264:893-920.

Huang Z, Satterlie RA (1990) Neuronal mechanisms underlying behavioral switching in a pteropod mollusc. J Comp Physiol [A] 166:875-887.

Karabelas AB, Purpura DP (1980) Evidence for autapses in the substantia nigra. Brain Res 200:467-473.

Kosaka T, Nagatsu I, Wu JY, Hama K (1986) Use of high concentrations of glutaraldehyde for immunocytochemistry of transmittersynthesizing enzymes in the central nervous system. Neuroscience 18:975-990.

Lalli CM (1970) Structure and function of the buccal apparatus of Clione limacina (Phipps) with a review of feeding in gymnosomatous pteropods. J Exp Mar Biol Ecol 4:101-118.

Litvinova NM, Orlovsky GN (1985) Feeding behavior of the pteropod mollusc Clione limacina. Bull Mosc Soc Nat Sect Biol 90:73-77 (in Russian).

Loechner KJ, Kaczmarek LK (1994) Autoactive peptides act at three distinct receptors to depolarize the bag cell neurons of Aplysia. J Neurophysiol 71:195-203.

Lubke J, Markram H, Frotscher M, Sakmann B (1996) Frequency and dendritic distribution of autapses established by layer 5 pyramidal neurons in the developing rat neocortex: comparison with synaptic innervation of adjacent neurons of the same class. J Neurosci 16:3209-3218.

Mercuri NB, Calabresi P, Stefani A, Stratta F, Bernardi G (1991) GABA depolarizes neurons in the rat striatum: an in vivo study. Synapse 8:38-40.

Michelson HB, Wong RKS (1991) Excitatory synaptic responses mediated by $\mathrm{GABA}_{\mathrm{A}}$ receptors in the hippocampus. Science 253:1420-1423.

Norekian TP (1993) Cerebral neurons underlying prey capture movements in the pteropod mollusc, Clione limacina. II. Afterdischarges. J Comp Physiol [A] 172:171-181.

Norekian TP, Satterlie RA (1993a) Cerebral neurons underlying prey capture movements in the pteropod mollusc, Clione limacina. I. Physiology, morphology. J Comp Physiol [A] 172:153-169.

Norekian TP, Satterlie RA (1993b) FMRFamide and GABA produce functionally opposite effects on prey-capture reactions in the pteropod mollusc Clione limacina. Biol Bull 185:248-262.

Norekian TP, Satterlie RA (1995) An identified cerebral interneuron initiates different elements of prey capture behavior in the pteropod mollusc, Clione limacina. Invert Neurosci 1:235-248.

Ogata N (1987) $\gamma$-Aminobutyric acid (GABA) causes consistent depolarization of neurons in the guinea pig supraoptic nucleus due to an absence of $\mathrm{GABA}_{\mathrm{B}}$ recognition sites. Brain Res 403:225-233.

Park MR, Lighthall JW, Kitai ST (1980) Recurrent inhibition in the rat neostriatum. Brain Res 194:359-369.

Pfeiffer-Linn C, Glantz RM (1989) Acetylcholine and GABA mediate opposing actions on neuronal chloride channels in crayfish. Science 245:1249-1251.

Pouzat C, Marty A (1998) Autaptic inhibitory currents recorded from interneurones in rat cerebellar slices. J Physiol (Lond) 509:777-783.

Preston RJ, Bishop GA, Kitai ST (1980) Medium spiny neuron projection from the rat striatum: an intracellular horseradish peroxidase study. Brain Res 183:253-263.

Segal MM (1991) Epileptiform activity in microcultures containing one excitatory hippocampal neuron. J Neurophysiol 65:761-770.

Segal MM (1994) Endogenous bursts underlie seizure-like activity in solitary excitatory hippocampal neurons in microcultures. J Neurophysiol 72:1874-1884.

Shi WX, Rayport S (1994) GABA synapses formed in vitro by local axon collaterals of nucleus accumbens neurons. J Neurosci 14:4548-4560.

Tamás G, Buhl EH, Somogyi P (1995) High degree of self-innervation 
by GABAergic basket cells in the visual cortex of the cat as revealed in vitro. J Physiol (Lond) 487:63P.

Tamás G, Buhl EH, Somogyi P (1997) Massive autaptic self-innervation of GABAergic neurons in cat visual cortex. J Neurosci 17:6352-6364.

ter Maat A, Geraerts WPM, Jansen RF, Bos NPA (1988) Chemically mediated positive feedback generates long-lasting afterdischarge in a molluscan neuroendocrine system. Brain Res 438:77-82.

Thompson SH, Smith SJ (1976) Depolarizing afterpotentials and burst production in molluscan pacemaker neurons. J Neurophysiol 39:153-162.

Van der Loos H, Glaser EM (1972) Autapses in neocortex cerebri: synapses between a pyramidal cell's axon and its own dendrites. Brain Res 48:355-360.

Vaney DI (1991) Many diverse types of retinal neurons show tracer coupling when injected with biocytin or neurobiotin. Neurosci Lett 125:187-190.

Wagner N (1885) Die Wirbellosen des Weissen Meeres: Zoologische Forschungen an der Kuste des Solowetzkischen Meerbusens in den Sommermonaten der Jahre. Leipzig, Germany: Verlag Von Wilhelm Engelmann.

White RL, Gardner D (1981) Self-inhibition alters firing patterns of neurons in Aplysia buccal ganglia. Brain Res 209:77-93.

Woodward RM, Polenzani L, Miledi R (1993) Characterization of bicuculline/baclofen-insensitive ( $\rho$-like) $\gamma$-aminobutyric acid receptors expressed in Xenopus oocytes. II. Pharmacology of $\gamma$-aminobutyric acidA and $\gamma$-aminobutyric acidB receptor agonists and antagonists. Mol Pharmacol 43:609-625. 